\title{
Fe-Rich Ferromagnetic Wires for Mechanical-Stress Self-Sensing Materials
}

\author{
Iñigo Liberal, Student Member, IEEE, Iñigo Ederra and Ramón Gonzalo, Member, IEEE
}

\begin{abstract}
The possibility of using Fe-rich wires in mechanical stress self-sensing materials is investigated. To this end, a retrieval technique aimed to characterize the high-frequency magnetoimpedance effect in ferromagnetic wires under mechanical stresses is proposed. The technique is based on the measurement of the wires inside a metallic rectangular waveguide, and it is validated through numerical simulations and tested with already published experimental data. In addition, the studied Fe-rich wires are characterized by the occurrence of the natural ferromagnetic resonance, whose frequency position increases from $7 \mathrm{GHz}$ to $8.25 \mathrm{GHz}$ for elongations ranging from $0 \mu \mathrm{m}$ to $60 \mu \mathrm{m}$.
\end{abstract}

Index Terms-Ferromagnetic Wires, Magnetoimpedance, Magnetic Materials, Mechanical-Stress Sensing, Composite Materials

\section{INTRODUCTION}

The magnetoimpedance (MI) effect on a ferromagnetic wire can be defined as the modulation of the electric current flowing along the wire due to changes in its magnetization state [1]-[4]. This general definition includes any alteration of the magnetization, such as changes in the size and shape of the magnetization domains, as well as changes in the magnetization direction and strength on a single domain. In view of the significant impedance changes produced by weak magnetic fields in the low-frequency range, the MI effect is usually regarded as giant magnetoimpedance (GMI) [5].

Technological applications of the MI effect have been traditionally related to the field of sensing devices in the lowfrequency range (up to $\mathrm{MHz}$ ) [6]. As a matter of fact, MI based sensors in which the ferromagnetic wire is integrated in an electronic circuit have been popular for applications not only including very sensitive magnetic field sensors, but also mechanical stress, temperature, position, chemical and multifunctional sensors [7].

More recently, sensing applications of ferromagnetic wires in the $\mathrm{GHz}$ frequency range have been proposed under the paradigm of self-sensing materials [8]. Instead of integrating the wires in an electronic circuit, an ensemble of wires is distributed within the sample under study, and the variations in the magnitude of interest are recorded as alterations in the reflected/transmitted fields when the sample is illuminated by an antenna. Because the MI effect is dominant, the ensemble of wires can be considered as an artificial dielectric material whose effective permittivity is a function of the MI effect [9]-[13]. This paradigm offers the possibility of a non-invasive/non-destructive inspection of mechanical stress and fracture, of great interest for architectural and health monitoring.
To the best knowledge of the authors, previous experiments on ferromagnetic wires for self-sensing materials have been focused on Co-rich wires [14]-[19]. Most probably this is due to the heritage of low-frequency MI sensors, in which the largest impedance variations are achieved with negative but near-zero magnetostriction constants. Contrarily, Fe-rich wires feature positive and large magnetostriction constants, which results in a dominant axial magnetization and in the presence of the natural ferromagnetic resonance (NFMR) at $\mathrm{GHz}$ frequencies [20], [21]. Therefore, Fe-rich wires are advantageous for highfrequency sensors, since no biasing field is required to produce the ferromagnetic resonance (FMR). Even in the presence of a biasing field, Fe-rich wires provide a stronger and higherfrequency FMR due to their higher magnetization at saturation, thus leading to higher spatial resolutions and smaller antennas. Moreover, a high magnetostriction constant ensures a strong response to external mechanical stresses.

This paper investigates the possibility of using Fe-rich wires for mechanical stress self-sensing materials, including the characterization of their high-frequency MI effect and the estimation of the effective permittivity of the resultant selfsensing material.

Note that the characterization of the wire impedance at highfrequencies can be a cumbersome task. As a matter of fact, while the wire impedance can be easily measured at the lowfrequency limit with an oscilloscope by means of the two/four probe method [22], as frequency increases the probe effect becomes dramatic and the wires tend to radiate. Therefore, high-frequency MI measurements are based on the integration of ferromagnetic wires in transmission lines, such as microstrip lines [14]-[16], coaxial cables [23], [24] and waveguides [19].

It is worth remarking that these experiments measure the impedance of the transmission line formed by the wire, and an additional retrieval technique is required to recover the actual wire impedance. Although this might seem a subtle difference, it must be considered for the design of self-sensing materials, since the wires are not integrated in any circuit/tranmission line, and thus the actual wire impedance is required to estimate the performance of the material.

The correct retrieval procedure for coaxial lines was presented in [23]. However, in that experiment both ends of the ferromagnetic wire must be connected to the line, which makes it difficult to apply mechanical stresses. To go further in the state of the art and overcome this problem, a retrieval procedure using metallic rectangular waveguides is presented here, enabling the evaluation of the high-frequency MI effect under mechanical stresses, and the consequent estimation of the self-sensing materials performance. 


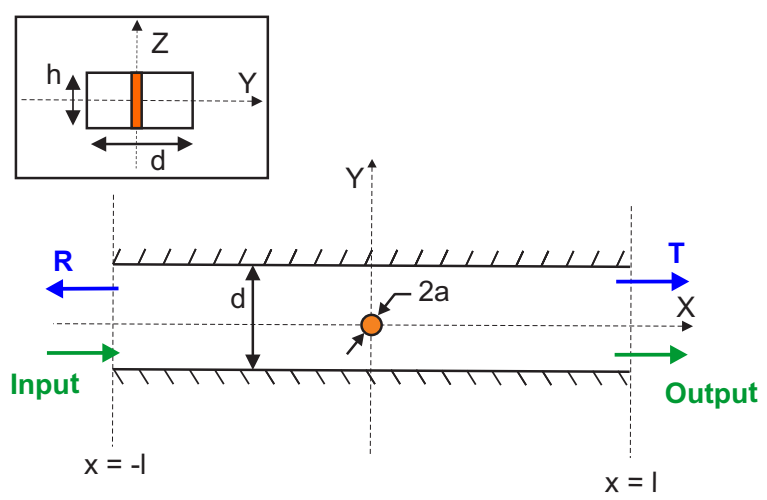

Fig. 1. Sketch of a ferromagnetic wire of radius $a$ inside a rectangular waveguide of width $d$, height $h$ and length $2 l$.

In addition, experiments on rectangular waveguides have been recently carried out to investigate the absorption spectrum of ferromagnetic wires [25] as well as their application in double negative materials [26]-[29]. Therefore, there is a collection of experimental data that could be analyzed with the proposed retrieval procedure, increasing the knowledge on the high-frequency MI effect.

In summary, this work introduces two main contributions: Firstly, Section II presents an experimental technique aimed to characterize the high-frequency MI effect under mechanical stresses, where numerical simulations are employed to validate the technique, and it is also applied to already available experimental data. Secondly, Fe-rich wires are evaluated as mechanical stress self-sensing materials in Section III, including the retrieval of the wire impedance and the prediction of the effective permittivity of the correspondent self-sensing materials.

\section{Retrieval PRocedure}

\section{A. Retrieval Procedure}

Fig. 1 represents the setup for the characterization of ferromagnetic wires in a metallic rectangular waveguide: a wire of radius $a$ is positioned in the middle of a waveguide of width $d$, height $h$ and length $2 l$. The wire ends are short-circuited to the waveguide walls to emulate an analytically tractable infinitely-long wire, and the incident electric field is parallel to the wire so that its magnetic response is excited. The goal of the retrieval procedure is to recover the wire impedance, $Z_{\mathrm{w}}$, from the reflection, $R$, and transmission, $T$, coefficients measured at the input and output of the waveguide.

This experimental setup has been analyticaly solved in [30], where closed-forms of the $R$ and $T$ coefficients have been formulated (see Eqs. 30 and 31 of the reference). Let us rewrite such equations to serve our experimental purposes: Firsly, the $R, T$ coefficients are formulated as a function of $Z_{\mathrm{w}}$ noting that the scattering coefficient, $a_{0}^{s}$, is given by $\left(a_{0}^{s}\right)^{-1}=-\frac{4}{k d}\left(Z_{\mathrm{w}}+\alpha_{0}^{-1}\right)$ [31]. Secondly, the $R, T$ coefficients are normalized to the amplitude of the electric field, and referred to the $x=-l$ and $x=l$ positions, respectively, so that they correspond to the measured $S_{11}$ and $S_{21}$ scattering parameters. By doing so, the $R, T$ coefficients can be written as

$$
\begin{gathered}
R=-\frac{\eta k}{k_{g} d} \cdot \frac{1}{Z_{\mathrm{w}}+\alpha_{0}^{-1}+\frac{\eta k}{4} G_{\mathrm{wg}}} e^{-j 2 k_{g} l} \\
T=\left[1-\frac{\eta k}{k_{g} d} \cdot \frac{1}{Z_{\mathrm{w}}+\alpha_{0}^{-1}+\frac{\eta k}{4} G_{\mathrm{wg}}}\right] e^{-j 2 k_{g} l}
\end{gathered}
$$

where $\eta=\sqrt{\mu_{0} / \epsilon_{0}}$ is the vacuum medium impedance, $k^{2}=\omega^{2} \mu_{0} \epsilon_{0}$ and $k_{g}^{2}=k^{2}-\left(\frac{\pi}{d}\right)^{2}$ are the free-space and the waveguide propagation constants, respectively. $\alpha_{0}^{-1}$ is the susceptibility of a perfect electric conductor (PEC) wire, given by [32]

$$
\alpha_{0}^{-1}=\frac{\eta k}{4}\left\{1+j \frac{2}{\pi}\left[\ln \left(\frac{2}{k a}\right)-0.5772\right]\right\}
$$

and $G_{\mathrm{wg}}$ is the interaction constant with the waveguide walls, given by [30]

$$
G_{\mathrm{wg}}=\sum_{n \neq 0} H_{0}^{(2)}(k|n 2 d|)-\sum_{n=-\infty}^{\infty} H_{0}^{(2)}(k|n 2 d-d|)
$$

where $H_{0}^{(2)}(-)$ is the Hankel function of the second kind and zero-th order.

In view of (1) and (2), it is straightforward to compute $Z_{\mathrm{w}}$ from either the $R$ or $T$ coefficients as follows

$$
\begin{gathered}
Z_{\mathrm{w}}^{R}=-\frac{\eta k}{k_{g} d} \frac{1}{R e^{j 2 k_{g} l}}-\alpha_{0}^{-1}-\frac{\eta k}{4} G_{\mathrm{wg}} \\
Z_{\mathrm{w}}^{T}=-\frac{\eta k}{k_{g} d} \frac{1}{T e^{j 2 k_{g} l}-1}-\alpha_{0}^{-1}-\frac{\eta k}{4} G_{\mathrm{wg}}
\end{gathered}
$$

The first term of (5) and (6) must be understood as the impedance of a wire inside the waveguide. The second and third terms subtract from this impedance the loading produced by the waveguide walls $\left(\eta k / 4 \cdot G_{\mathrm{wg}}\right)$ and the impedance of a PEC wire $\left(\alpha_{0}^{-1}\right)$, so that the final result is the actual wire impedance, $Z_{\mathrm{w}}$.

In theory, $Z_{\mathrm{w}}^{R}=Z_{\mathrm{w}}^{T}=Z_{\mathrm{w}}$, so that $Z_{\mathrm{w}}$ can be indifferently computed from $R$ or $T$. From a practical standpoint, $R$ is significantly smaller than $T$ due to the reduced cross-section of the wire as compared to the waveguide width, and therefore $Z_{\mathrm{w}}^{R}$ is much more sensitive to noise, reflections produced by mismatched junctions and other experimental artefacts than $Z_{\mathrm{w}}^{T}$.

\section{B. Numerical Validation}

In order to check the accuracy of the analytical model employed for the retrieval technique, the results have been tested with a well-established full-wave electromagnetic solver: CST Microwave Studio [33].

The model of the numerical simulation is depicted in Fig. 2 and consists of a WR-90 metallic rectangular waveguide ( $d=22.86 \mathrm{~mm}, h=11 \mathrm{~mm}, 2 l=15 \mathrm{~cm})$ and a wire placed in the middle of the waveguide. The inset in the figure represents the wire geometry. Note that the simulation of a rectangular waveguide (of a few wavelengths size) with a ferromagnetic wire (of $10^{-4 \sim-5}$ wavelengths size) with finite conductivity and gyrotropic magnetic susceptibility is a cumbersome task 


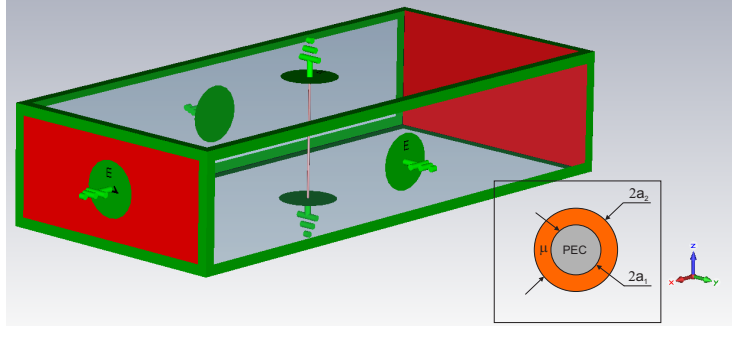

Fig. 2. CST Model of the WR-90 waveguide with connected wire. The inset in the figure represents the structure of the simulated wire.

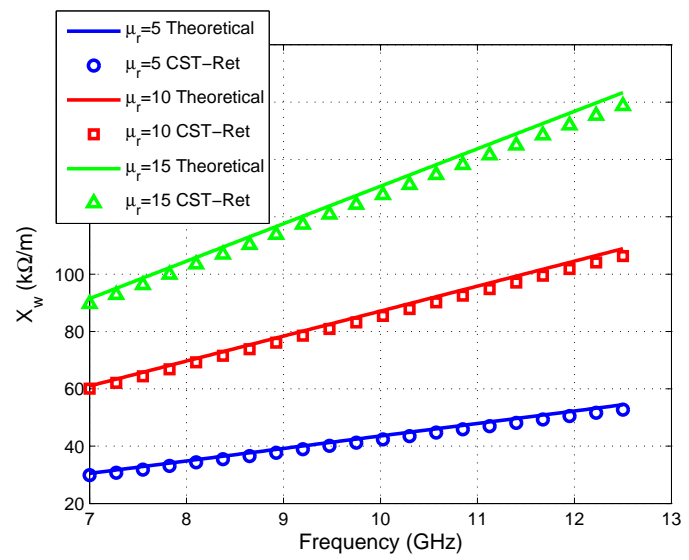

Fig. 3. Comparison of the theoretical wire reactance and the reactance retrieved from the $R, T$ coefficients simulated with CST

for current numerical solvers, and therefore the numerical simulations have been performed with a simpler, two layer wire. Such a wire is composed of an inner PEC wire of radius $a_{1}$, covered by a magnetic coating of permeability $\mu=\mu_{r} \mu_{0}$, and total radius $a_{2}$. The distributed impedance of this type of wires can be found as [34]

$$
Z_{\mathrm{w}}=j \frac{\omega \mu}{2 \pi} \cdot \ln \left(\frac{a_{2}}{a_{1}}\right)
$$

In essence, the magnetic coating loads the PEC wire with a given reactance (for lossless $\mu$ ), which increases with $\mu$ and the ratio between the external and internal radii, $a_{2} / a_{1}$.

Three different values of the magnetic coating $\mu_{r}=$ 5, 10 and 15, with dimensions $a_{2}=0.1 \mathrm{~mm}$ and $a_{1}=$ $0.05 \mathrm{~mm}$, have been employed in the numerical simulations. Once the $R, T$ coefficients have been computed with CST, $Z_{\mathrm{w}}$ has been determined by means of the proposed retrieval technique and compared to the theoretical solution (i. e. (7)), as it is shown in Fig. 3. In this numerical example, $Z_{\mathrm{w}}^{R}$ and $Z_{\mathrm{w}}^{T}$ converged perfectly, and therefore a single $Z_{\mathrm{w}}$ line has been represented. It can be concluded that there is a good agreement between the retrieved and theoretical results. Small differences can be ascribed to numerical errors in the simulator (mesh and truncation), the truncation of the $G_{\mathrm{wg}}$ interaction constant, (4), and the small argument bessel function approximations intrinsically assumed in the theoretical $Z_{\mathrm{w}},(7)$.

\section{Experimental Validation}

In [25], three different $\left(\mathrm{Co}_{0.94} \mathrm{Fe}_{0.06}\right)_{75} \mathrm{Si}_{12.5} \mathrm{~B}_{12.5}$ wires of $22.5 \mu \mathrm{m}, 5 \mu \mathrm{m}$ and $2 \mu \mathrm{m}$ metallic radius were measured by the authors in a metallic rectangular waveguide in order to investigate the correlation between the wire geometry and absorption spectrum. The wires were trapped between two WR-90 waveguides so that the experimental setup is similar to the one described in this paper for the retrieval technique (see Fig. 1). Thus, those experimental data can be analyzed to test the consistency of the retrieval technique. Since the wires are saturated by an external electromagnet, the retrieved impedances can be compared to the theoretical study of MI in saturated wires also presented by the authors in [31].

The retrieved $Z_{\mathrm{w}}$ are depicted in Fig. 4, where each color line corresponds to a different biasing magnetic field as in [25]. The results present the expected response of FMR in saturated wires: On the one hand, a peak of resistance appears at the FMR, whose frequency position increases along with the biasing field. In addition, the resistance increases as the wire radius decreases, due to the higher penetration of the electromagnetic field in the wires. On the other hand, the retrieved reactance presents the expected maximum-minimum sequence. However, asymmetric reactances produced by parasitic reactive loads can be appreciated in the figure. This parasitic reactive loading is an artifact of the experimental setup, produced by the small separation between the waveguides where wires are positioned. Note that the measurements were originally carried out to evaluate the absorption spectrum, in which this effect has a minor impact, and that it can be mitigated with the correct setup/calibration procedure.

\section{Fe-RICH Wires FOR SElF-SENSING Materials}

\section{A. Characterization of the Wires}

In order to assess the performance of Fe-rich wires as mechanical stress self-sensing materials, a $\mathrm{Fe}_{77.5} \mathrm{Si}_{12.5} \mathrm{~B}_{10}$ wire was fabricated by means of the Taylor-Ulitovsky technique [35], [36] at the Material Science Institute of Madrid (ICMM), Prof. M. Vázquez. A SEM image of the wire was taken at the Foundation for the Research and Development of Nanotechnology in Navarra (Fidena) and it is represented in Fig. 5. As it is shown, the wire consists of a metallic core of $5.25 \mu \mathrm{m}$ radius and total radius (metallic core and pyrex coating) of $12.5 \mu \mathrm{m}$.

A photograph of the experimental setup employed to characterize the wires is depicted in Fig. 6. To further clarify the setup, the figure includes schematic views of the setup in the XY-, XZ-, and YZ-planes. Following the technique introduced in Section II, the distributed impedance of the wires has been retrieved from the measurement of the $R$ and $T$ coefficients in a WR-112 rectangular waveguide $(d=28.6 \mathrm{~mm}$, $h=12.8 \mathrm{~mm}, 2 l=10 \mathrm{~cm}$ ). Both waveguide ends have been connected to SMA adaptors and the scattering parameters have been measured in an Agilent PNA-X N5242A network analyzer.

A small hole has been drilled in the center of the broad wall of the waveguide. By doing so, the wires can entirely cross the waveguide while being perpendicular to the incident electric 


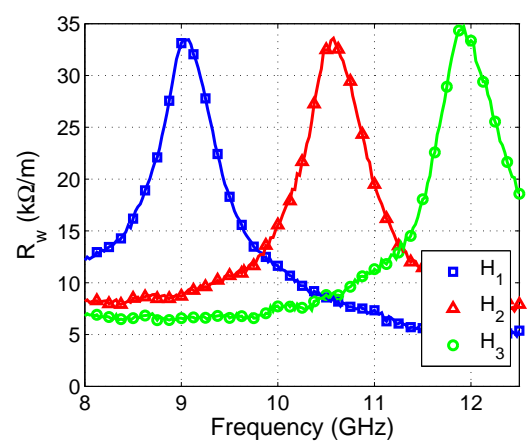

(a)

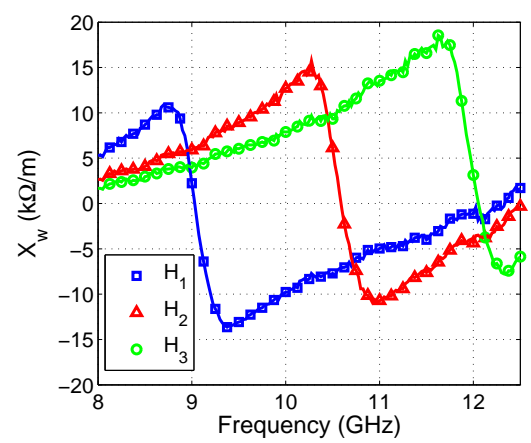

(d)

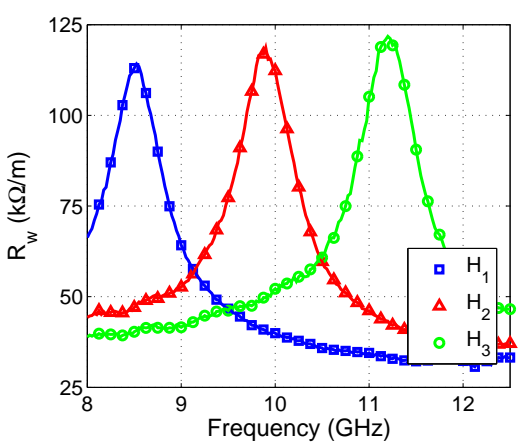

(b)

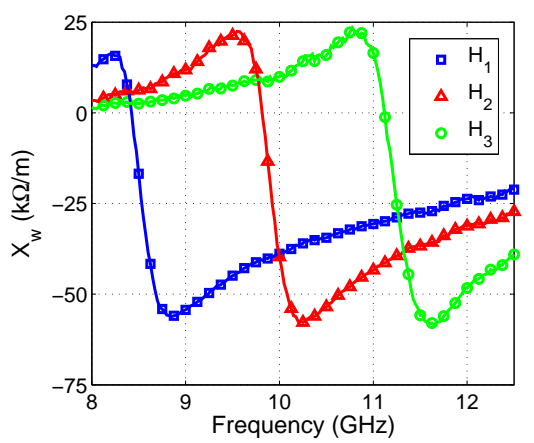

(e)

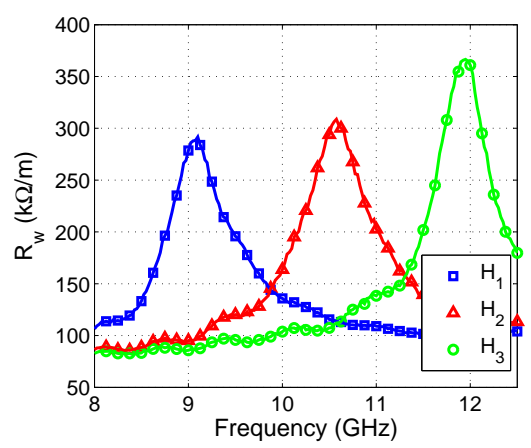

(c)

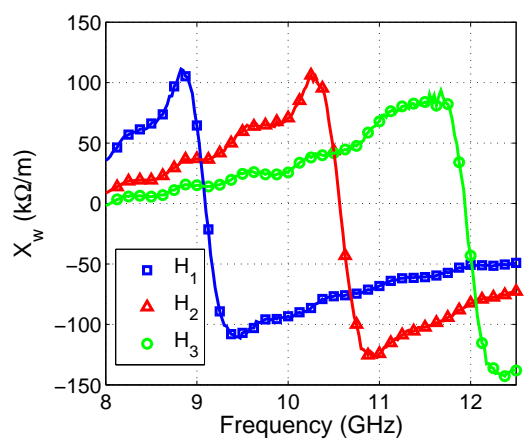

(f)

Fig. 4. Retrieved resistance (a)-(c) and reactance (d)-(f) for $\left(\mathrm{Co}_{0.94} \mathrm{Fe}_{0.06}\right)_{75} \mathrm{Si}_{12.5} \mathrm{~B}_{12.5}$ wires of $22.5 \mu \mathrm{m}, 5 \mu \mathrm{m}$ and $2 \mu \mathrm{m}$ metallic radius, respectively. Each line corresponds with a different biasing field $H_{1}=89.4 \mathrm{kA} / \mathrm{m}, H_{2}=117.7 \mathrm{kA} / \mathrm{m}$ and $H_{3}=146.0 \mathrm{kA} / \mathrm{m}$, directed along the wire axis.

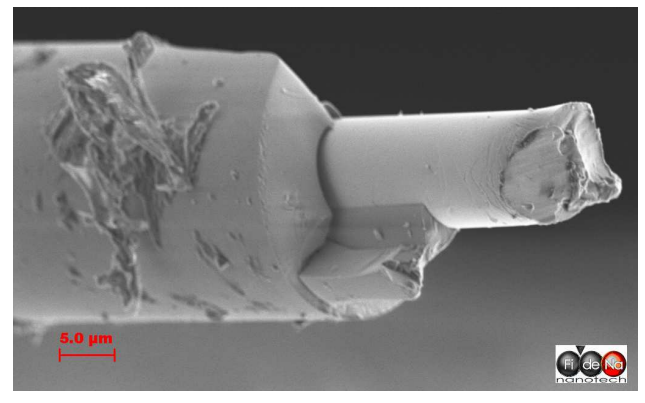

Fig. 5. SEM image of the Fe-rich wire under test.

field, i.e. the wires are positioned along the Y-axis according to Fig. 6. Due to the orientation of the waveguide, no holder or insulating platform is required.

Outside the waveguide, both wire ends have been fixed to micropositioners. In this way, when one of the micropositioners is shifted mechanical stresses are produced along the wires axis, resulting in an elongation $d L$. Due to the magnetostrictive behavior of the wires, such mechanical stresses result in variations on their magnetic, which are recorded as variations in the $R$ and $T$ coefficients, and thus on the retrieved distributed impedance. It is worth remarking that no external DC magnetic field has been employed to polarize the ferromagnetic wires.

The retrieved distributed impedance $Z_{\mathrm{w}}$ as a function of the wire elongation is represented in Fig. 7. It can be concluded that the wires feature the typical resonant behavior produced by the NFMR. Namely, a maximum of resistance and an inductive-to-capacitive transition around the resonance.
In addition, the elongation of the wires from 0 to $60 \mu \mathrm{m}$ produces a shift of the NFMR frequency, $f_{\mathrm{NFMR}}$ from $7 \mathrm{GHz}$ to $8.25 \mathrm{GHz}$.

This behavior is ascribed to an increase in the anisotropy field, $H_{k}$. When no mechanical forces are applied to the wire, $H_{k}$ is defined by the mechanical stresses produced during the fabrication process. Due to the positive magnetostriction constant of the wires, these stresses result in a positive $H_{k}$ and the occurrence of the NFMR. Therefore, additional mechanical stresses increase $H_{k}$, with the consequent increase in $f_{\mathrm{NFMR}}$. To further clarify this fact, note that $f_{\mathrm{NFMR}}$, is given by the well-known Kittel relationship [37]

$$
f_{\mathrm{NFMR}}=\frac{\mu_{0} \gamma}{2 \pi} \sqrt{H_{k}\left(H_{k}+M_{s}\right)}
$$

where $\gamma$ is the gyromagnetic ratio and $M_{s}$ is the magnetization at saturation. Inversely, for $M_{s} \gg H_{k}$ the anisotropy field can be determined as follows

$$
H_{k}=\left(\frac{2 \pi}{\mu_{0} \gamma}\right)^{2} \frac{f_{\mathrm{NFMR}}^{2}}{M_{s}}
$$

Fig. 8 depicts $H_{k}$ and $f_{\mathrm{NFMR}}^{2}$ as a function of the elongation in the wires. To this end, $f_{\mathrm{FMR}}$ has been fixed to the frequency of maximal resistance, and typical parameters of Fe-rich wires [20] $\mu_{0} M_{s}=1.60 \mathrm{~T}$ and $\gamma=2.088 \cdot 10^{11} T^{-1} s^{-1}$ have been assumed. It can be concluded that the retrieved values of $H_{k}$, approximately ranging from $20 \mathrm{kA} / \mathrm{m}$ to $30 \mathrm{kA} / \mathrm{m}$, are in accordance to those of wires with high magnetostriction constant [38]. Furthermore, both $H_{k}$ and $f_{\mathrm{FMR}}^{2}$ increase linearly along with the elongation. 

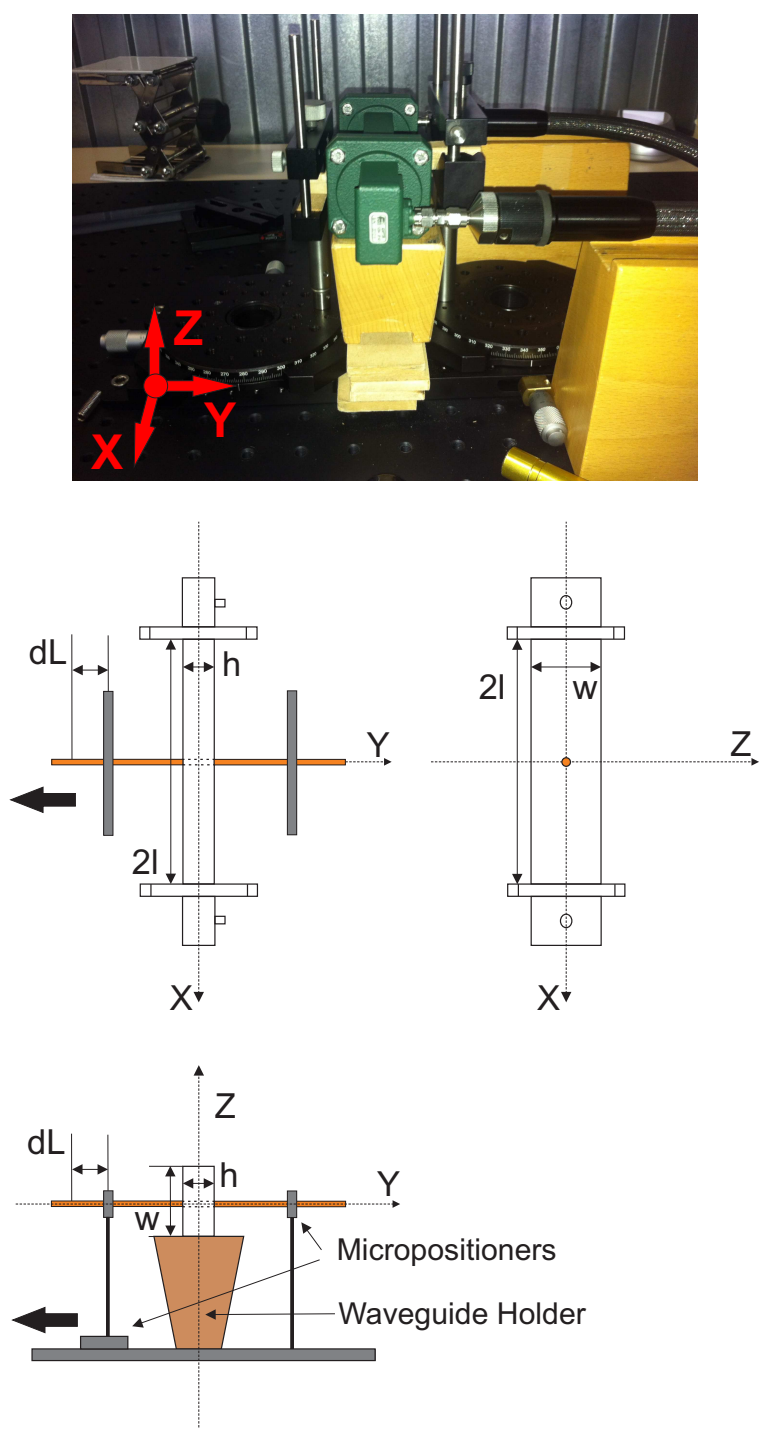

Fig. 6. Photograph of the experimental setup and schematic views in the $\mathrm{XY}-, \mathrm{XZ}-$, and YZ-planes. The black arrow shows the direction of the shift.

\section{B. Estimation of Material Response}

Once the wire impedance $Z_{\mathrm{w}}$ has been characterized, it is possible to estimate the performance of the resultant selfsensing materials through classical electromagnetic mixing formulas. Due to practical reasons, these composite materials typically consist of ensembles of short-wires, which result in artificial dielectric materials whose effective permittivity is defined by the electric dipole moments produced by the shortwires.

A short-wire electric polarizability can be determined through an antenna analogy, i. e. noting that a short-wire is equivalent to a receiving dipole antenna with a short-circuited feeding gap, and distributed impedance $Z_{\mathrm{w}}$. Therefore, assuming a short-dipole antenna $(L \ll \lambda)$ oriented along the $z$ direction with current distribution in the receiving mode $I(z)=I_{0}\left(1-z^{2} / L^{2}\right)$, the wire polarizability is given by [39]

$$
\alpha=\frac{1}{j \omega} \frac{L}{Z_{\text {in }}+\frac{2}{3} L Z_{\mathrm{w}}}\left[\frac{4}{3} L+\frac{2 L^{2}}{45} \frac{Z_{\mathrm{w}}}{Z_{\mathrm{inp}}}\right]
$$

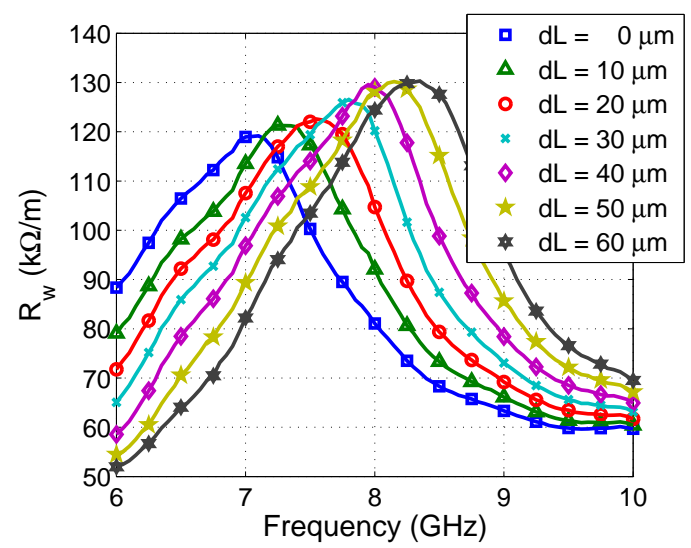

(a)

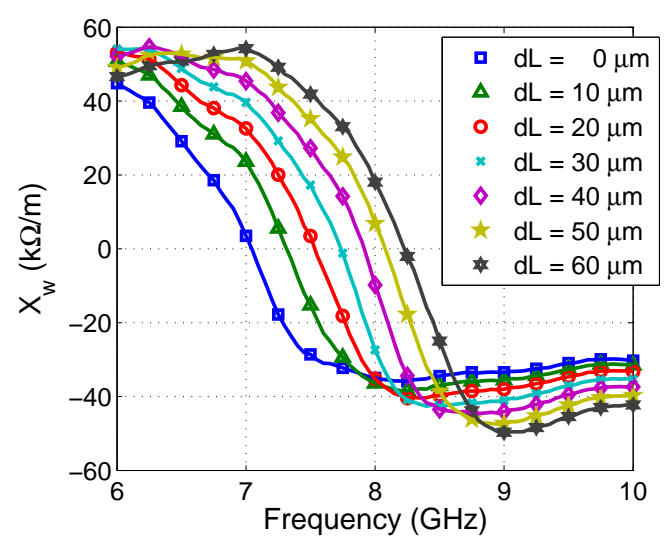

(b)

Fig. 7. (a) Resistance and (b) reactance of a $\mathrm{Fe}_{77.5} \mathrm{Si}_{12.5} \mathrm{~B}_{10}$ wire of metallic radius $a=5.25 \mu \mathrm{m}$ for different $d L$ elongation values.

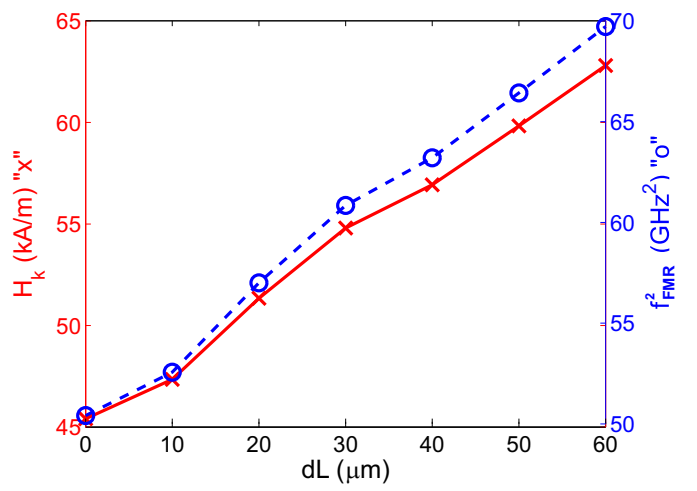

Fig. 8. Anisotropy field $H_{k}$ and square of the FMR frequency $f_{\mathrm{FMR}}^{2}$ as a function of the wire elongation, $d L$.

where $Z_{\text {in }}$ is the input impedance of the reciprocal transmitting antenna, and $2 L$ is the length of the wire.

Knowing the polarizability of a given inclusion, it is straightforward to apply mixing rules to estimate the effective permittivity. For the sake of simplicity, let us start with a mixture of wires aligned along the $z$ axis. Under a MaxwellGarnett formalism, it is known that an aligned mixture of ellipsoids produces an effective uniaxial permittivity [40]

$$
\overline{\overline{\epsilon_{\text {eff }}}}=\epsilon_{x} \hat{\mathbf{x}} \hat{\mathbf{x}}+\epsilon_{y} \hat{\mathbf{y}} \hat{\mathbf{y}}+\epsilon_{z} \hat{\mathbf{z}} \hat{\mathbf{z}}
$$


where each of its elements is given by

$$
\epsilon_{i}=\epsilon_{h}+\frac{p \alpha_{i}}{1-N_{i} \frac{p \alpha_{i}}{\epsilon_{h}}} \quad i=x, y, z
$$

$\epsilon_{h}$ is the polarizability of the host medium, $p$ is the number of wires per unit of volume, and $N_{i}$ and $\alpha_{i}$ are the depolarization factor and the polarizability of the ellipsoid along the $i$ direction.

Due to the high aspect ratio of the wires the "needle" depolarization factors $N_{x}=N_{y}=\frac{1}{2}, N_{z}=0$ are the natural choice, and it is sufficient to consider the polarizability along the wires (i.e. $\alpha_{x}=\alpha_{y}=0, \alpha_{z}=\alpha$ ). Therefore, the effective permittivity simplifies to

$$
\overline{\overline{\epsilon_{\mathrm{eff}}}}=\epsilon_{h}(\hat{\mathbf{x}} \hat{\mathbf{x}}+\hat{\mathbf{y}} \hat{\mathbf{y}})+\epsilon_{z} \hat{\mathbf{z}} \hat{\mathbf{z}}
$$

with

$$
\epsilon_{z}=\epsilon_{h}+p \alpha
$$

Fig. 9(a)-(b) represents the computed real and imaginary parts of the z-component of the effective permittivity $\epsilon_{z} / \epsilon_{0}$ for wires of length $L=1 \mathrm{~mm}$ and density $p=5$ wires $/ \mathrm{cm}^{3}$. It can be appreciated that the real part of the effective permittivity is positive as in mixtures of conductive short wires, although its dipersion profile is affected by the NFMR. Furthermore, the composite is lossy with maximal losses below the NFMR, as noted in [25].

As expected, external stresses shift the permittivity dispersion profile towards higher frequencies, which can be measured through variations in the reflection/transmission from the sample, or directly from the retrieval of its constitutive parameters. If only a part of the sample is subjected to mechanical stresses, it must be treated as an heterogeneous solid, where the shape and size of the volume affected by the mechanical stresses can be determined through inverse scattering techniques.

In most practical applications the mixture will be a random ensemble of wires. In this case the directionality of the mixtures vanishes and the sample behaves as an isotropic mixture

$$
\overline{\overline{\epsilon_{\mathrm{eff}}}}=\epsilon_{m}(\hat{\mathbf{x}} \hat{\mathbf{x}}+\hat{\mathbf{y}} \hat{\mathbf{y}}+\hat{\mathbf{z}} \hat{\mathbf{z}})
$$

where the electric polarization results from the averaging of each direction susceptibility [40]. For wires with high aspect ratio only one susceptibility component is relevant and therefore

$$
\epsilon_{m}=\epsilon_{h}+p \alpha / 3
$$

Fig. 9(c)-(d) shows the effective permittivity of the same mixture of wires than in Fig. 9(a)-(b), but with random orientation of the wires. The results confirm that the response of a random mixture is a diluted version of the aligned mixture.

Both random and aligned mixtures are subjected to the limitations of homogenization models. Therefore, their predictions will be accurate as long as the wires and the separation between wires is small enough, and as long as a sufficiently large number of wires is affected by the mechanical stresses. If the former condition is not fulfilled, the structure must be inevitability modeled with complex theory of groups of individual scatterers. If the latter conditions is not fulfilled, the structure can still be modeled as an homogeneous mixture, but multiphase mixing rules must be applied. In general, the Pyrex coating of the wires prevents the appearance of percolation processes in the mixtures. However, one must recall this effect when dealing with wires after glass-removal processes. In those cases, more generalized mixing formulas must be adopted [41].

To sum up, multiple models can be adopted to predict the performance of a self-sensing material, where the selection of the model depends on the properties of the mixture. In any case, all applicable models rely on the characterization of the wires through retrieval techniques, as discussed in previous sections.

\section{Conclusions}

This work has investigated the possibility of using $\mathrm{Fe}$ rich wires as mechanical stress self-sensing materials. In order to evaluate the performance of these wires, a retrieval technique aimed to evaluate the high-frequency MI effect from measurements in a rectangular waveguide has been introduced. Furthermore, the waveguide can be perforated to enable the application of mechanical stresses upon the wire under study. The retrieval technique consists of a simple analytical formulation, which has been validated through numerical simulations in a full-wave electromagnetic solver. In addition, the available literature on measurements of ferromagnetic wires inside a rectangular waveguide can be processed with the proposed retrieval technique, providing new experimental data about the MI effect at high frequencies.

A further extension of this work might include the derivation of retrieval techniques for other popular experimental setups, for example, resonant cavities. Thus, it will be possible to analyze the available experimental data based on those experimental setups, and to compare the performance of difference setups.

To evaluate the performance of Fe-rich wires as self-sensing materials, a $\mathrm{Fe}_{77.5} \mathrm{Si}_{12.5} \mathrm{~B}_{10}$ wire of $a=5.25 \mu \mathrm{m}$ metallic radius has been characterized with the proposed retrieval technique. The wire main feature is the presence of the FMR at $7 \mathrm{GHz}$ with no magnetic biasing. Moreover, the magnetostrictive behaviour of the wires leads to shifts in the FMR frequency from $7 \mathrm{GHz}$ to $8.25 \mathrm{GHz}$ for elongations ranging from 0 to $60 \mu \mathrm{m}$. Finally, it has been pointed out how to proceed to the estimation of the corresponding self-sensing materials performance, identifying potential scenarios such as aligned and random mixtures of wires, non-homogenizable disperse mixtures of wires and multiphase mixtures of wires.

\section{ACKNOWLEDGMENT}

This work was supported by the Spanish Ministry of Science and Innovation, Dirección General de Investigación y Gestión del Plan Nacional de I+D+I, Subdirección General de Proyectos de Investigación, Project Nos. TEC2009-11995 and CSD2008-00066.

The authors thank Prof. M. Vázquez of the Material Science Institute of Madrid (ICMM) for providing the ferromagnetic wires, and J. Bravo of the Foundation for the Research 


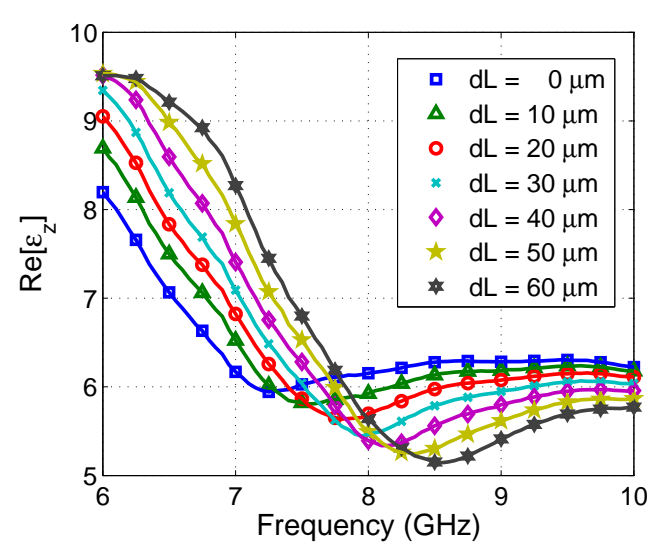

(a)

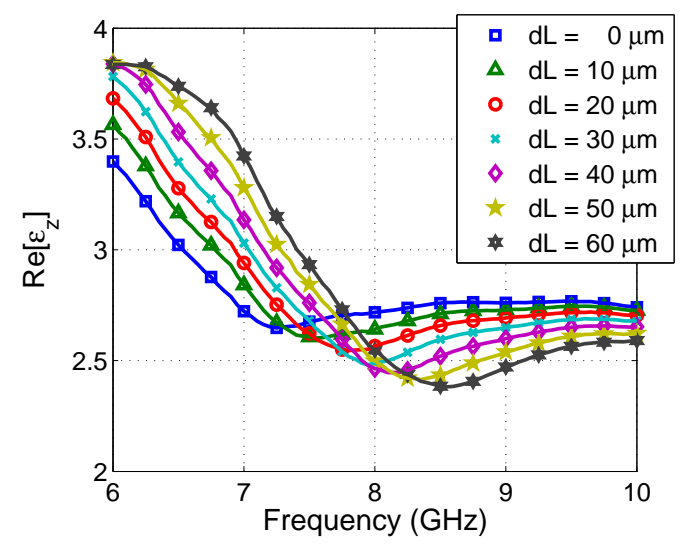

(c)

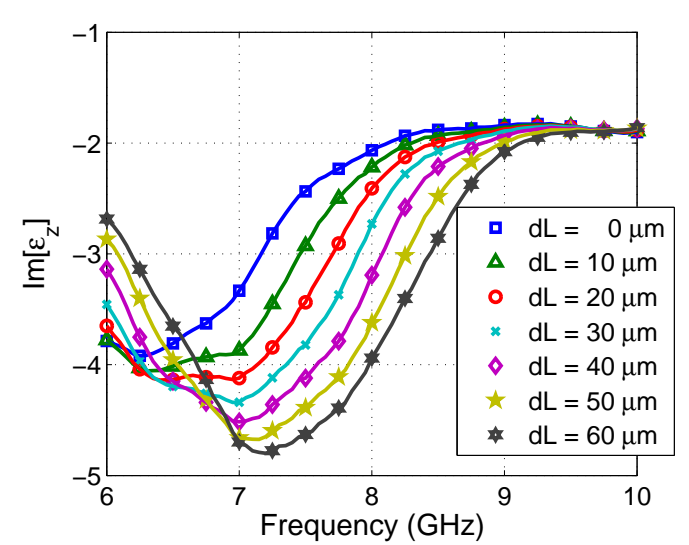

(b)

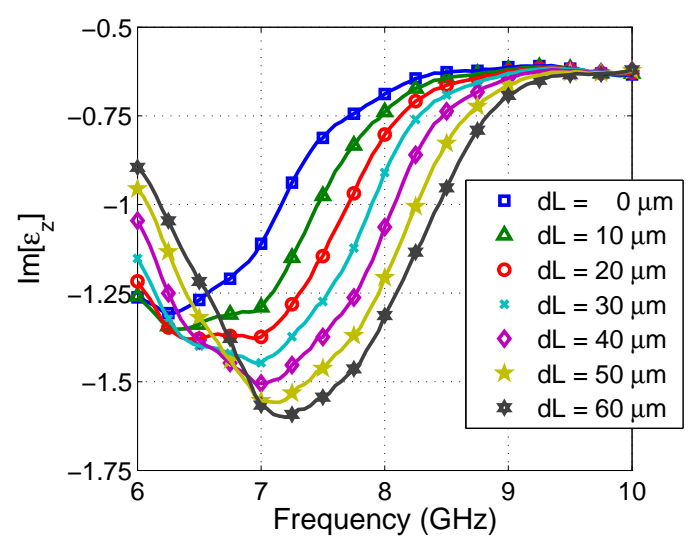

(d)

Fig. 9. Real and imaginary parts of the effective permittivity for aligned (a)-(b) and random (c)-(d) mixtures of short $(L=1$ mm) Fe-rich wires with density $p=5$ wires $/ \mathrm{cm}^{3}$ as a function of the wire elongation, $d L$.

and Development of Nanotechnology in Navarra (Fidena) for taking the SEM image.

\section{REFERENCES}

[1] L. V. Panina, K. Mohri, K. Bushida, and M. Noda, "Giant magnetoimpedance and magneto-inductive effects in amorphous alloys (invited)," Journal of Applied Physics, vol. 76, no. 10, p. 6198, 1994.

[2] J. Velázquez, M. Vázquez, D. X. CHen, and A. Hernando, "Giant Magnetoimpedance in nonmagnetostrictive amorphous wires," Physical Review B, vol. 50, no. 22, pp. 737-740, 1994.

[3] D. Menard, M. Britel, P. Ciureanu, and A. Yelon, "Giant magnetoimpedance in a cylindrical magnetic conductor," Journal of Applied Physics, vol. 84, no. 5, p. 2805, 1998.

[4] M. Knobel, M. L. Sánchez, C. Gómez-Polo, P. Marín, M. Vázquez, and A. Hernando, "Giant magneto-impedance effect in nanostructured magnetic wires," Journal of Applied Physics, vol. 79, no. 3, pp. 1646-1654, 1996.

[5] K. V. Rao, F. B. Humphrey, and J. L. Costa-Kramer, "Very large magneto-impedance in amorphous soft ferromagnetic wires," Journal of Applied Physics, vol. 76, no. 10, p. 6204, 1994.

[6] M. Vázquez, M. Knobel, M. L. Sánchez, R. Valenzuela, and A. P. Zhukov, "Giant magnetoimpedance effect in soft magnetic wires for sensor applications," Sensors and Actuators A: Physical, vol. 59, no. 1-3, pp. 20-29, Apr. 1997.

[7] J. Torrejón, G. Badini Confalonieri, K. Pirota, and M. Vázquez, "Multifunctional magnetoelastic sensor device based in multilayer magnetic microwires," Sensor Letters, vol. 5, no. 1, pp. 153-156, 2007.

[8] D. P. Makhnovskiy and L. V. Panina, "Field and Stress-Tunable Microwave Composite Materials Based on Ferromagnetic Wires," in Progress in Ferromagnetism Research, V. N. Murray, Ed. HauppaugeNY: Nova Science Publishers, Inc, 2005, pp. 257-295.
[9] - "Field dependent permittivity of composite materials containing ferromagnetic wires," Journal of Applied Physics, vol. 93, no. 7, p. 4120, 2003.

[10] O. Reynet, A. Adenot-Engelvin, S. Deprot, O. Acher, and M. Latrach, "Effect of the magnetic properties of the inclusions on the highfrequency dielectric response of diluted composites," Physical Review $B$, vol. 66, no. 9, pp. 1-9, Sep. 2002.

[11] D. P. Makhnovskiy and L. V. Panina, "Experimental demonstration of tunable scattering spectra at microwave frequencies in composite media containing $\mathrm{CoFeCrSiB}$ glass-coated amorphous ferromagnetic wires and comparison with theory," Physical Review B, vol. 74, no. 6, pp. 1-11, Aug. 2006.

[12] I. Liberal, I. S. Nefedov, I. Ederra, R. Gonzalo, and S. A. Tretyakov, "On the effective permittivity of arrays of ferromagnetic wires," Journal of Applied Physics, vol. 110, no. 10, p. 104902, 2011.

[13] L. V. Panina, M. Ipatov, V. Zhukova, A. Zhukov, and J. Gonzalez, "Magnetic field effects in artificial dielectrics with arrays of magnetic wires at microwaves," Journal of Applied Physics, vol. 109, no. 5, p. 053901, 2011.

[14] L. V. Panina, S. I. Sandacci, and D. P. Makhnovskiy, "Stress effect on magnetoimpedance in amorphous wires at gigahertz frequencies and application to stress-tunable microwave composite materials," Journal of Applied Physics, vol. 97, no. 1, p. 013701, 2005.

[15] M. Ipatov, A. Chizhik, V. Zhukova, J. Gonzalez, and A. Zhukov, "Correlation of surface domain structure and magneto-impedance in amorphous microwires," Journal of Applied Physics, vol. 109, no. 11, p. 113924, 2011.

[16] M. Ipatov, V. Zhukova, A. Zhukov, J. Gonzalez, and A. Zvezdin, "High frequency magneto impedance in amorphous microwires," Journal of Physics: Conference Series, vol. 200, no. 8, p. 082009, Jan. 2010.

[17] F. Qin, C. Brosseau, and H. X. Peng, "In situ microwave characterization of microwire composites under mechanical stress," Applied Physics Letters, vol. 99, no. 25, p. 252902, 2011. 
[18] F. X. Qin, N. Pankratov, H. X. Peng, M. H. Phan, L. V. Panina, M. Ipatov, V. Zhukova, A. Zhukov, and J. Gonzalez, "Novel magnetic microwiresembedded composites for structural health monitoring applications," Journal of Applied Physics, vol. 107, no. 9, p. 09A314, 2010.

[19] F. X. Qin, H. X. Peng, L. V. Panina, M. Ipatov, V. Zhukova, A. Zhukov, and J. Gonzalez, "Smart Composites With Short Ferromagnetic Microwires for Microwave Applications," IEEE Transactions on Magnetics, vol. 47, no. 10, pp. 4481-4484, 2011.

[20] A. Zhukov, M. Vázquez, J. Velázquez, A. Hernando, and V. Larin, "Magnetic properties of fe-based glass-coated microwires," Journal of Magnetism and Magnetic Materials, vol. 170, no. 3, pp. 323 - 330, 1997.

[21] A. Zhukov, A. Cobeo, J. Gonzalez, A. Torcunov, E. Pina, M. Prieto, J. Blanco, V. Larin, and S. Baranov, "Ferromagnetic resonance, magnetic behaviour and structure of fe-based glass-coated microwires," Journal of Magnetism and Magnetic Materials, vol. 203, no. 1?3, pp. 238 - 240, 1999.

[22] K. Mandal, S. Puerta, M. Vázquez, and A. Hernando, "The frequency and stress dependence of giant magnetoimpedance in amorphous microwires," IEEE Transactions on Magnetics, vol. 36, no. 5, pp. 32573259, 2000.

[23] D. Menard, M. Britel, P. Ciureanu, a. Yelon, V. P. Paramonov, a. S. Antonov, P. Rudkowski, and J. O. Strom-Olsen, "High frequency impedance spectra of soft amorphous fibers," Journal of Applied Physics, vol. 81 , no. 8, p. 4032, 1997.

[24] J. Torrejón, G. a. Badini-Confalonieri, and M. Vázquez, "Multipeak ferromagnetic resonance behaviour tailored by magnetoelastic coupling in FeSiB/CoNi layered microwires," Journal of Physics D: Applied Physics, vol. 43, no. 14, p. 145001, Apr. 2010.

[25] I. Liberal, I. Ederra, C. Gómez-Polo, A. Labrador, J. Pérez-Landazabal, and R. Gonzalo, "A Comprehensive Analysis of the Absorption Spectrum of Ferromagnetic Wires," "In press" IEEE Transactions Microwave Theory and Techniques, DOI: 10.1109/TMTT.2012.2195022, 2012.

[26] J. Carbonell, H. García-Miquel, and J. Sánchez-Dehesa, "Double negative metamaterials based on ferromagnetic microwires," Physical Review $B$, vol. 81 , no. 2, pp. 1-6, Jan. 2010.

[27] A. Labrador, C. Gómez-Polo, J. Pérez-Landazabal, V. Zablotskii, I. Ederra, R. Gonzalo, G. Badini-Confalonieri, and M. Vázquez, "Magnetotunable left-handed FeSiB ferromagnetic microwires." Optics letters, vol. 35 , no. 13 , pp. 2161-2163, Jul. 2010.

[28] H. García-Miquel, J. Carbonell, and J. Sánchez-Dehesa, "Left handed material based on amorphous ferromagnetic microwires tunable by $\mathrm{dc}$ current," Applied Physics Letters, vol. 97, no. 9, p. 094102, 2010.

[29] H. García-Miquel, J. Carbonell, V. E. Boria, and J. Sánchez-Dehesa, "Experimental evidence of left handed transmission through arrays of ferromagnetic microwires," Applied Physics Letters, vol. 94, no. 5, p. 054103, 2009.

[30] I. Liberal, I. Ederra, C. Gómez-Polo, A. Labrador, J. Pérez-Landazabal, and R. Gonzalo, "Theoretical Modeling and Experimental Verification of the Scattering From a Ferromagnetic Microwire," IEEE Transactions on Microwave Theory and Techniques, vol. 59, no. 3, pp. 517-526, 2011.

[31] I. Liberal, I. S. Nefedov, I. Ederra, R. Gonzalo, and S. A. Tretyakov, "Electromagnetic response and homogenization of grids of ferromagnetic microwires," Journal of Applied Physics, vol. 110, no. 6, p. 064909 , 2011.

[32] P. A. Belov, C. R. Simovski, and S. A. Tretyakov, "Two-dimensional electromagnetic crystals formed by reactively loaded wires," Physical Review E, vol. 66, no. 3, pp. 6610-6616, Sep. 2002.

[33] CST, Computer Simulation Technology. [Online]. Available: http://www.cst.com/

[34] H. Strifors and G. Gaunaurd, "Scattering of electromagnetic waves by a perfectly conducting cylinder with a thin lossy magnetic coating," IEEE Transactions on Antennas and Propagation, vol. 48, no. 10, pp. 15281532, 2000.

[35] G. Taylor, "A Method of Drawing Metallic Filaments and a Discussion of Their Properties and Uses," Physical Review, vol. 23, no. 5, pp. 655660, 1924.

[36] A. Ulitovski and N. Avernin, "Method of fabrication of metallic microwire," p. 14, 1964.

[37] A. G. Gurevich and G. A. Melkov, Magnetization Oscillations and Waves. CRC Press, New York, 1996.

[38] S. E. Lofland, H. García-Miquel, M. Vázquez, and S. Bhagat, "Microwave magnetoabsorption in glass-coated amorphous microwires with radii close to skin depth," Journal of Applied Physics, vol. 92, no. 4, p. 2058, 2002.
[39] S. A. Tretyakov, S. Maslovski, and P. A. Belov, "An analytical model of metamaterials based on loaded wire dipoles," IEEE Transactions on Antennas and Propagation, vol. 51, no. 10, pp. 2652-2658, Oct. 2003.

[40] A. Sihvola, Electromagnetic Mixing Formulas and Applications. The Institution of Engineering and Technology, London, 1999.

[41] D. S, McLachlan, A. Priou, I. Chenerie, E. Issac, and F. Henry, "Modeling the permittivity of composite materials with a general effective medium equation," Journal of Electromagnetic Waves and Applications, vol. 6, pp. $1099-1131,1992$.

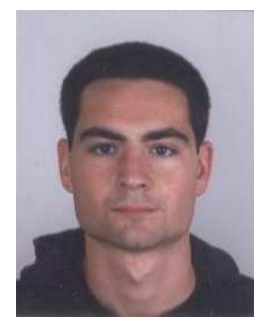

Iñigo Liberal was born on October 31, 1985 in Pamplona, Navarra, Spain. In 2009 he received the M. Sc. Degree in Telecommunication Engineering from the Public University of Navarra (UPNA), Pamplona, Spain. He was the recipient of a scholarship to carry out his M. Sc. Thesis at the International Research Center for Telecommunications and Radar (IRCTR) at the Delft University of Technology, The Netherlands.

Since August 2009 he has been with the Antenna Group at the Public University of Navarra (UPNA), where he is currently working towards his Ph.D. His main research interests are in the field of high-frequency applications of magnetic materials, the design of low-profile metamaterial-inspired antennas and UWB antenna systems.

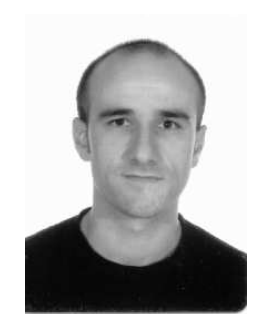

Iñigo Ederra was born in Isaba, Navarra, Spain in 1972. He received the Ingeniero de Telecomunicación and Ph.D. degrees from the Universidad Pública de Navarra, Pamplona, Spain, in 1996 and 2004, respectively.

In 1997, he joined the Microwave and Millimetre Wave Group, Universidad Pública de Navarra. From 1999 to 2000 he was with the European Space Research and Technology Centre (ESTEC), ESA, Noordwijk, The Netherlands, where he was working on Electromagnetic Bandgap materials and their applications in the field of antennas. Since 2001 he is with the Antenna Group, Universidad Pública de Navarra. From June to October 2002 he was visitor scientist at the Rutherford Appleton Laboratory, Chilton, Didcot, UK, participating in the Startiger project.

He was co-recipient of the LAPC 2006 and IWAT 2007 best paper award. His research interests are in the field of Electromagnetic Bandgap materials and Metamaterials and their applications in microwave and millimetre wave components and antennas. 


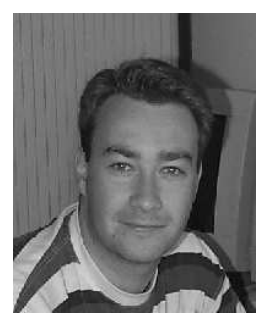

Ramón Gonzalo (S 95) was born on July 15, 1972 in Logroño, La Rioja. He received the M.Sc. and the $\mathrm{Ph}$. D. degree in Ingeniero de Telecomunicación both with honors, from the Public University of Navarra (UPNa), Spain.

Since October 1995 he is with the Antennas Group at the Electrical and Electronic Engineering Department in UPNa where he currently is Associate Professor. From September 1997 to December 1998, he was joined as Research Fellow to the Antenna Section in ESA-ESTEC where he was involved in the modelling and design of electromagnetic crystal devices at microwave and millimetre wave frequencies. He has been involved in more than 25 research project, European, and National level acting as co-ordinator in several of them. In particular he has been co-ordinator of 5 projects funded by the European Space Agency, 2 projects in the framework of the European Commission and several of them funded by the Spanish Minister of Science. He has more than 50 journal publications in peer review magazines and 100 conference papers related to his research lines. From January 2006 untill April 2008 he was acting as sub-director of the Engineering Faculty and from April 2008 he is acting as Head of Electrical and Electronic Engineering Department.

His current area of research is in the field of Terahertz technologies, subsystems and devices, Electromagnetic Band Gap technology with emphasis on space antenna applications, design of waveguide transmission lines and corrugated horn antennas. 